\title{
Snap-Through Instability Patterns in Truss Structures
}

\author{
Glenn A. Hrinda* \\ NASA Langley Research Center, Hampton, Virginia 23831
}

\begin{abstract}
Geometrically nonlinear truss structures with snap-through behavior are demonstrated by using an arc length approach within a finite element analysis. The instability patterns are equilibrium paths that are plotted throughout the snap-through event. Careful observation of these patterns helps to identify weak designs in large space structures, as well as identify desirable snap-through behavior in the miniaturization of electronic devices known as microelectromechanicalsystems (MEMS). Examples of highly nonlinear trusses that show snap-through behavior are examined by tracing their equilibrium paths.
\end{abstract}

\section{Nomenclature}

dof $\quad=$ degree-of-freedom

$\mathrm{lb} \quad=$ pound

MEMS = microelectromechanicalsystems

\section{Introduction}

T arge highly flexible truss systems have been investigated in the past to support new space technologies. Examples include solar power supply constellations that use trusses as their main support structure, deployable reticulated trusses for mounting solar array panels (Fig. 1) and large space truss structures for supporting microwave antennae and mirrors. ${ }^{1}$ Flexible trusses are well suited for space structures because of their compact packaging in the launch vehicle, their lighter mass and their reduced deployment time once in orbit. Understanding the large elastic displacement of these types of structures can prevent sudden buckling failures from applied operational and construction loads (Fig. 2). The structures must also be designed to maintain their integrity even after an initial inelastic failure. Some structures may collapse after a localized failure of one of their members. This is a progressive failure that redistributes loads, which causes other members to fail until the total structure becomes unstable. ${ }^{2}$ Slight manufacturing or assembly defects in the truss geometry can increase the risk for an unstable structure. The truss defects may also compromise the load-carrying capability of the structure. ${ }^{3}$ In this work, unique truss designs that exhibit snap-through instability are investigated. Their complex equilibrium paths are explained to inform engineers of possible nonlinear behavior in designs and that instability may occur before a design bifurcation limit is reached.

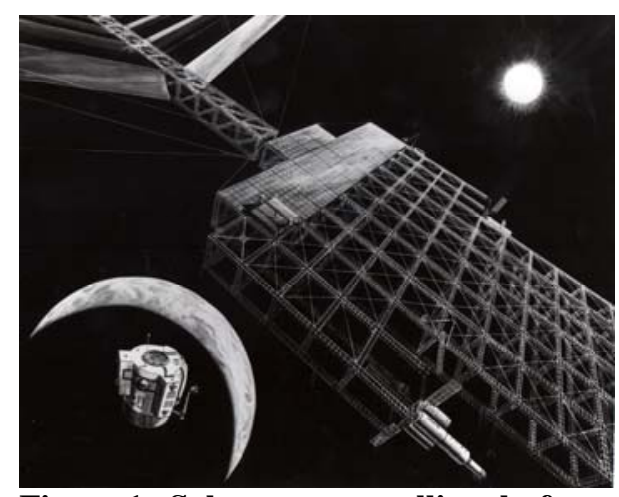

Figure 1. Solar power satellite platform.

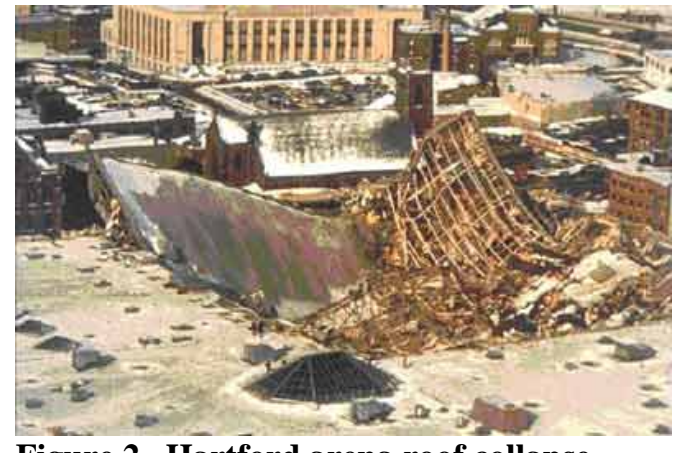

Figure 2. Hartford arena roof collapse.

Research Engineer, NASA Langley Research Center, AIAA member. 
Another current use for the snap-through structure is in the miniaturization of electronic devices known as microelectromechanicalsystems (MEMS). ${ }^{4}$ These are formed microstructures that are integrated into silicon chips. The microstructures may be formed by trusses or plates that snap through under electrostatic forces. Figure 3 shows a MEMS switch before and after snap through. The micro switch, a silicon beam that is approximately 25 micrometers in length, is activated by an electrostatic charge.

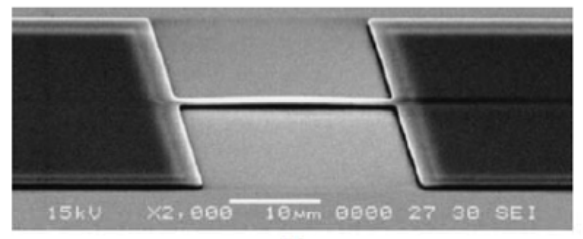

(a)

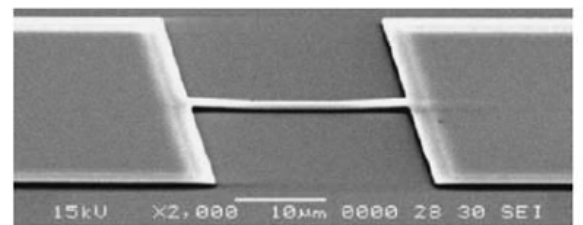

(b)

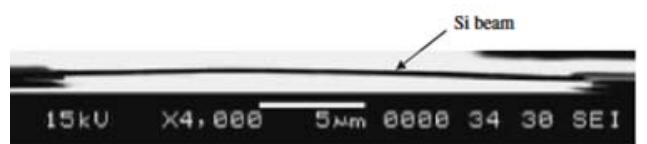

(a)

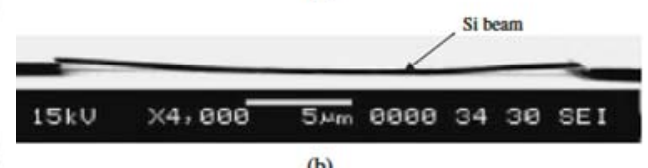

(b)

Figure 3. Photographs of fabricated MEMS switch (a) Before snap-through, (b) After snap-through. ${ }^{4}$

\section{Objectives}

The primary goal of this investigation is to define truss geometries that exhibit complex nonlinear snap-through behavior. One type of truss configuration that is prone to snap-through is the shallow truss structure. This type of structure loses its stability differently than a structure that is undergoing bifurcation buckling. Finite element programs typically solve for elastic stability by running an eigenvalue buckling solution. However, shallow truss structures typically lose their ability to support any load well below their bifurcation load limit. If a standard eigenvalue buckling solution is run in NASTRAN for a shallow truss structure, then the load that is predicted in the analysis may be much higher than the snap-through load that is predicted with the arc length solver in NASTRAN.

Another goal is to understand why highly flexible structures may support loads up to a limit point and then suddenly snap through to a new stable equilibrium state while still remaining elastic. The post snap-through geometry may support an additional load up to the design limit of the structure, or it can suddenly snap back. An understanding of this nonlinear behavior can be gained by plotting the equilibrium path. The graphic trace of the equilibrium path is the load versus the displacement at a particular point on the structure. An accurate plot of the path shows the sensitivity of the truss response to load changes.

\section{Analysis}

A stable truss design exists when deformations increase as the applied load increases; an unstable design occurs when deformations increase as the load decreases. To understand this type of structural response, the equilibrium paths of key connection points are plotted. A typical equilibrium plot is shown in Fig. 4 . The shallow truss geometry prompts snap-through buckling of many members rather than local buckling of a single member. Here a shallow truss is defined as a truss system that has a small out-of-plane dimension over a large span. The nonlinear buckling nature of shallow trusses includes a post-buckling instability region along the equilibrium path, which is shown in Fig. 4. The nonlinear instability region is the region in which "snap-through" occurs and in which the equilibrium path goes from one stable point (1) to another new stable point (2). The nonlinear behavior places the critical limit load at point 1 equal to that at point 2, but the load limit corresponds to a new structural shape. The second stable point along the equilibrium path occurs after a large displacement of the structure. During this snap-through, the slope of the equilibrium path (load versus deflection) eventually becomes zero. The slope of this curve is also referred to as the "tangent stiffness. ${ }^{5}$ When the tangent stiffness softens and approaches zero for a single-degree-offreedom system, many nonlinear solvers encounter convergence problems. Some solvers immediately jump to point 2 without identifying the unstable snap-through path. Bifurcation buckling is also shown in Fig. 4 with a linear, prebuckling region along the equilibrium path up to a critical load point $\left(P_{\text {critical }}\right)$. At the bifurcation point the structure 
immediately becomes unstable and buckles. The member is unable to support any additional load, which is not the case for nonlinear snap-through buckling. The focus of this work is on the fundamental path rather than the bifurcation points. The inertial forces during snap-through are not addressed in this work; the focus is confined to the application of static methods.

Five truss designs are used to investigate the snap-through buckling behavior. All of the models demonstrate an attempt to find a nonlinear equilibrium path that is similar to that shown in Fig. 4. The designs are shallow trusses that have critical limit points. The designs are related to those from Khot and Kamat $^{6}$, Crisfield $^{7,8}{ }^{\text {,Hrinda }}{ }^{9}$ and are reviewed in detail in Ref. 10. The designs are analyzed by using a finite element approach and solved by using NASTRAN's nonlinear buckling solution. The results are plotted to form the system's nonlinear equilibrium trace. The dynamic inertial effects along the unstable region along the equilibrium path are excluded. The dynamic effects that result from the slender nature of the truss members are minimal at snapthrough. The first shallow truss solves a four-element asymmetric system with a vertical load applied at the midpoint and pinned-end conditions. The next truss problem is an eight-element system with equal lengths that resembles a spoke wheel. Then, a larger 16-member shallow truss problem is presented, which is similar to the Crisfield star dome that is discussed in volume two of Ref. 8. This problem was analyzed by using a constant cross-sectional area and long slender members. The fourth truss problem is a symmetric A-truss that is loaded at the apex node. The last truss problem is the large, two-dimensional arch truss that was used by Crisfield ${ }^{8}$.

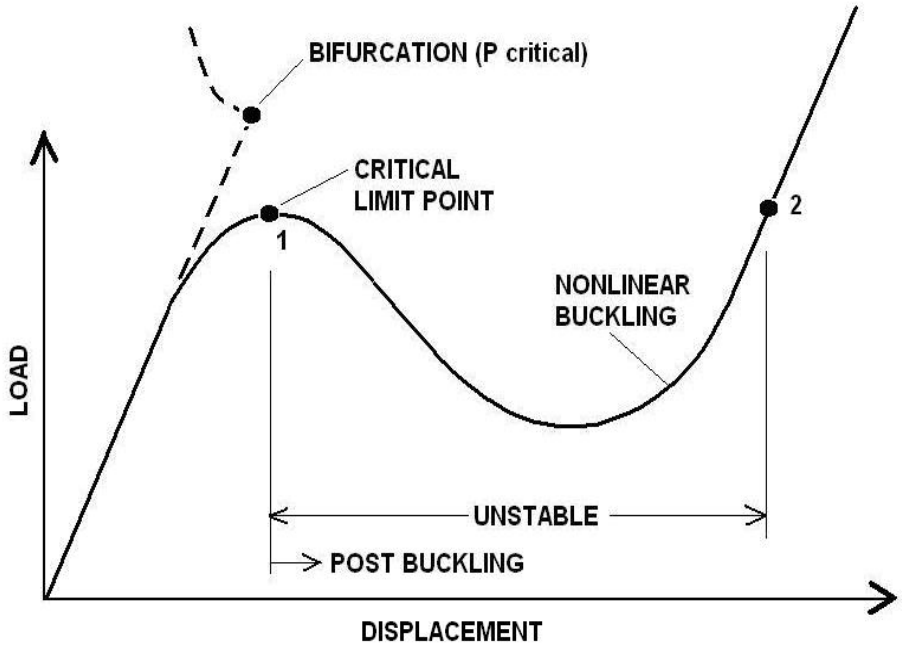

Figure 4. Equilibrium paths for nonlinear and bifurcation buckling.

\section{A. Four-Member Asymmetric Truss}

The first problem, shown in Fig. 5, is a four-member asymmetric truss with five nodes. The outer nodes are located 100 in. along the $x$ axis from the center node and 20 in. along the $y$ axis. The center node is raised 20 in. along the $z$ axis to create the shallow truss. A $10000 \mathrm{lb}$ vertical load is applied at the center node to cause snapthrough. The cross-sectional area of all members is $1 \mathrm{in}^{2}$ with a Young's modulus of $10 \mathrm{e}^{6} \mathrm{lb} / \mathrm{in}^{2}$.

The results from NASTRAN are shown by the displacement plots in Fig. 6 and the load-displacement plot in Fig. 7. The plot shows the equilibrium path of the unconstrained center node. The four points that appear next to the curve correspond to the data that are listed sequentially in Table 1. The critical limit, which occurs at point 1 in Fig. 7, is reached at a load increment of 0.56 and a center node displacement of -8.66 in. Here, the structure becomes unstable and suddenly snaps through to point 4 . The geometry undergoes a large nonlinear displacement without material yielding. Between points 1 and 4, the load increment reverses twice as the displacements continue to increase. When the load increment reaches -0.56 , which occurs at point 3 , the load reverses and increases until the final load. The plot shows that the critical limit load is the increment of 0.56 multiplied by
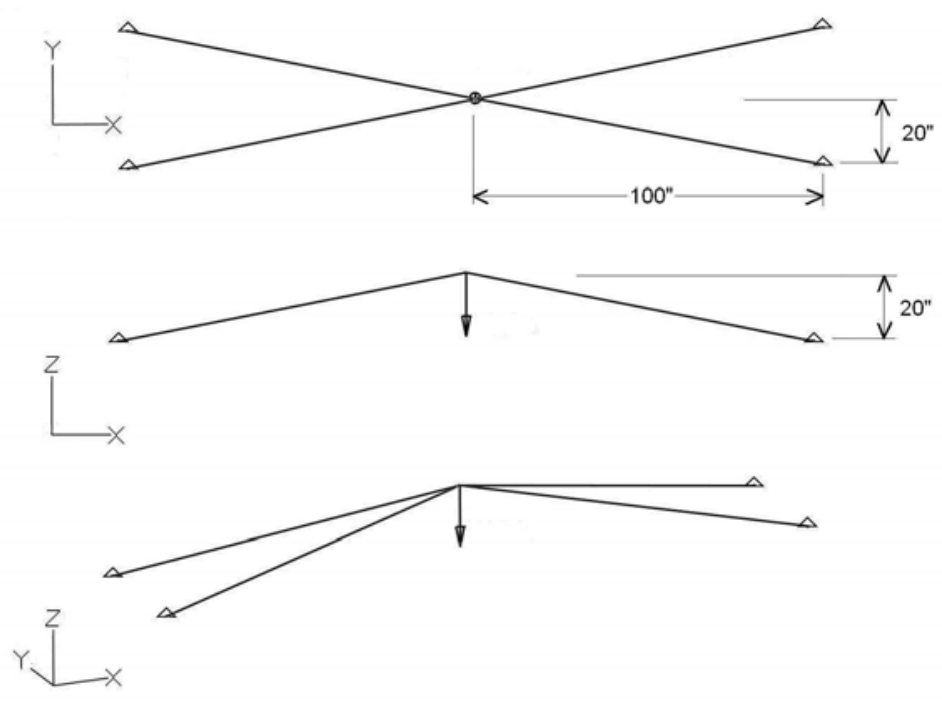

Figure 5. Four-member asymmetric truss example. 
the applied load of $10000 \mathrm{lb}$. This critical limit load is equal to $5600 \mathrm{lb}$ and is the maximum load that the structure can support before snap-through. As shown in Fig. 7, the second stable point can be located by extending from point 1 a vertical line that intersects the equilibrium path at point 4 . At this point, the dynamic snap-through stops and the structure is now able to support additional loading.
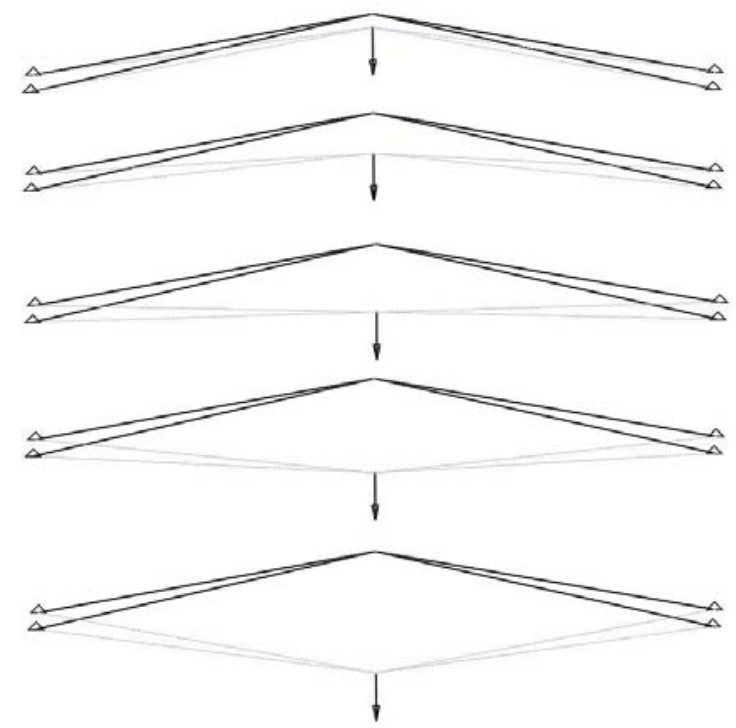

Table 1. Four-Member Truss Example

\begin{tabular}{|c|c|c|}
\hline Location & Load increment & $\begin{array}{c}\text { Displacement, } \\
\text { inches }\end{array}$ \\
\hline 1 & 0.56 & -8.66 \\
\hline 2 & 0.00 & -19.89 \\
\hline 3 & -0.56 & -31.39 \\
\hline 4 & 0.56 & -43.17 \\
\hline
\end{tabular}

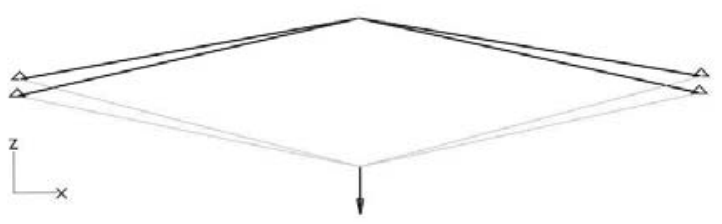

Figure 6. Four-member asymmetric truss displacement.

Load Increment

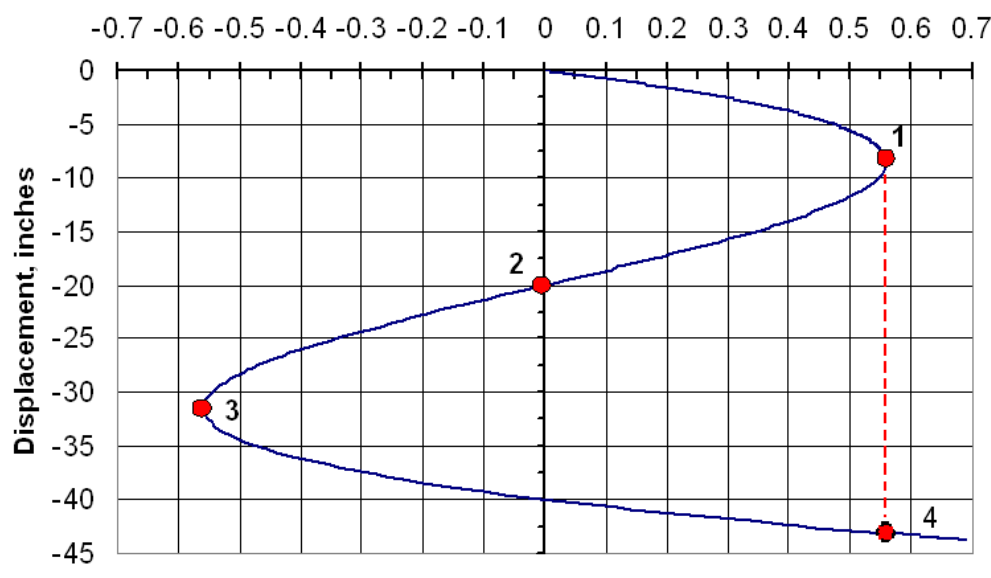

Load vs. Displacement

Figure 7. Four-member asymmetric truss equilibrium path during snap-through. 


\section{B. Eight-Member Symmetric Shallow Truss}

The next example is an eight-member symmetric shallow truss which is shown in Fig. 8. Each member has a horizontal length of 500 in. measured from the center node. The vertical out-of-plane dimension of the common center node is $40 \mathrm{in}$. All members have an area of $10 \mathrm{in}^{2}$ and a Young's modulus of $10000 \mathrm{lb} / \mathrm{in}^{2}$. A downward vertical $1000 \mathrm{lb}$ load is applied at the center node to allow snap-through. The eight outer nodes are constrained with pin supports with the center node left unconstrained.

The displacement of the truss is shown in Fig. 9 and the equilibrium path of the center node is plotted in Fig. 10. The load increment is increased until a critical limit load of 0.078 is reached at a displacement of -16.75 in. This occurs at point 1 in Fig. 10 and corresponds to the first set of plot data that is given in Table 2. As in the first example, this model also suddenly snaps through to a new reconfigured stable geometry. The structure is unstable along the equilibrium path from points 1 through 4 . After point 1 , the displacements continue to increase as the load increment decreases. At data point 3, the load increment decreases to -0.078 and immediately reverses. The equilibrium path continues to point 4 , which is the next stable equilibrium point of the structure. The dashed line in Fig. 10 shows the range of unstable displacements and indicates the next stable equilibrium location, which is point 4 . The final displacement is 115.24 in. at the final applied load increment of 0.96 . The equilibrium path that is shown demonstrates that the structure can support $78.3 \mathrm{lb}$ without snap-through buckling. The load is determined by multiplying the maximum load increment by the applied load of $1000 \mathrm{lb}$. After snapthrough, the structure becomes stable once again at point 4 and is able to support loads. The increase in loading begins at the same critical limit load that initially caused the snap-through.

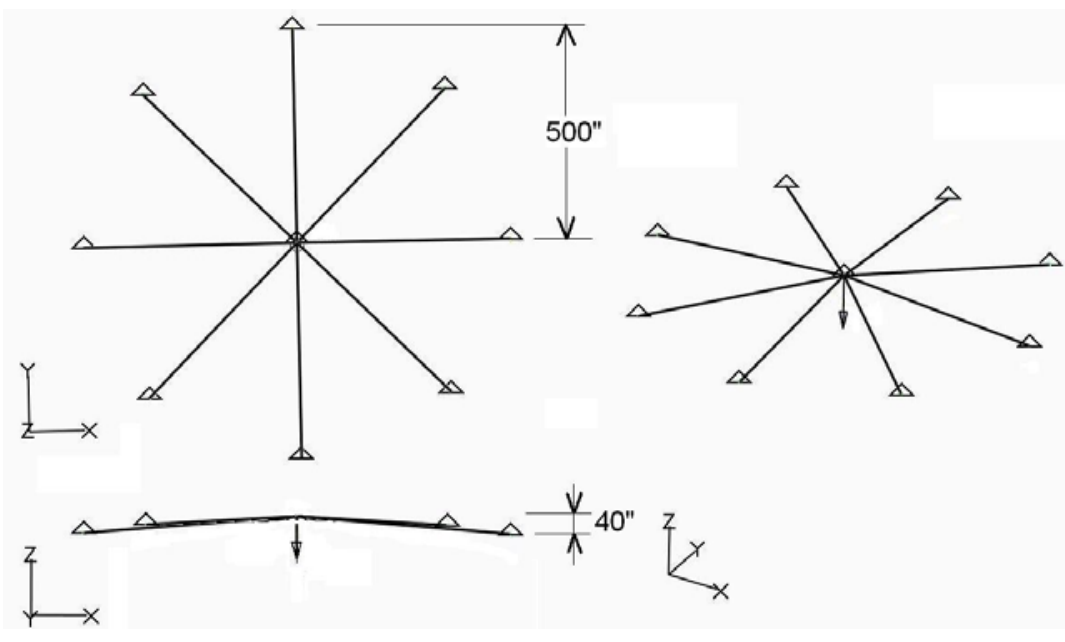

Figure 8. Eight-member truss example.
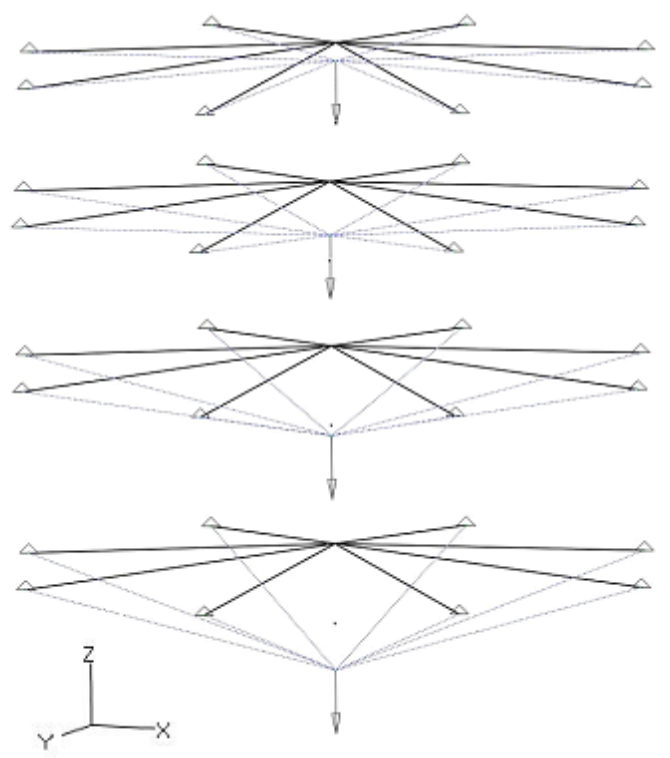

Table 2. Eight-Member Truss Example

\begin{tabular}{|c|c|c|}
\hline Location & Load Increment & $\begin{array}{c}\text { Displacement, } \\
\text { inches }\end{array}$ \\
\hline 1 & 0.0783 & -16.75 \\
\hline 2 & 0.00 & -40.85 \\
\hline 3 & -0.0783 & -62.76 \\
\hline
\end{tabular}

Figure 9. Eight-member truss displacements. 


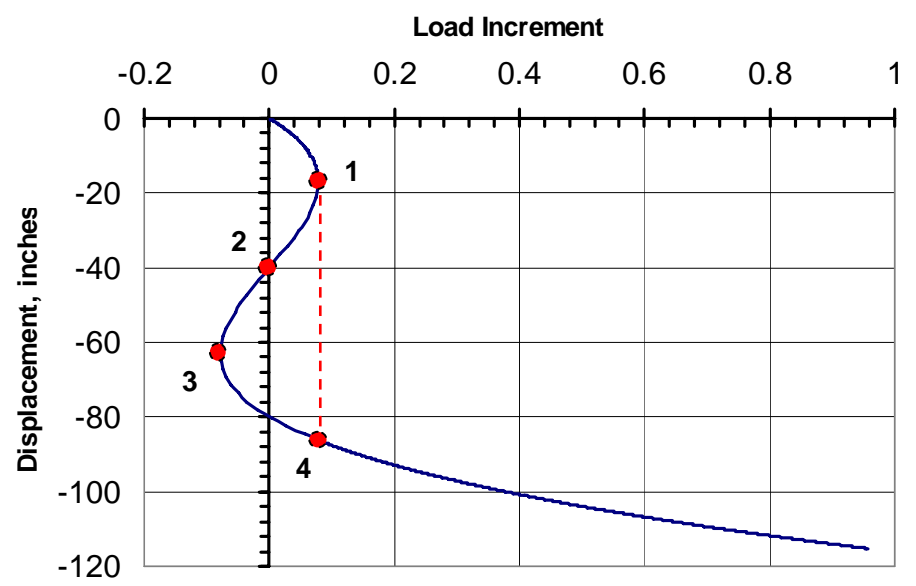

Load vs. Displacement

Figure 10. Eight-member truss equilibrium path.

\section{Sixteen-Member Shallow Truss}

The next model is a symmetric sixteen-element truss, which is shown in Fig. 11. The truss has nine nodes, with the four corner nodes constrained. The plan view of the structure gives the dimensions of the outer corner nodes. The vertical out-of-plane dimension, which places this design in the "shallow truss" family, is 4 in. The other nodes are spaced evenly between the outer nodes as shown. A $10^{6} \mathrm{lb}$ load is applied vertically at the center node to force the structure to buckle. The cross sectional area is $1 \mathrm{in}^{2}$ and the Young's modulus is $10^{7} \mathrm{lb} / \mathrm{in}^{2}$.

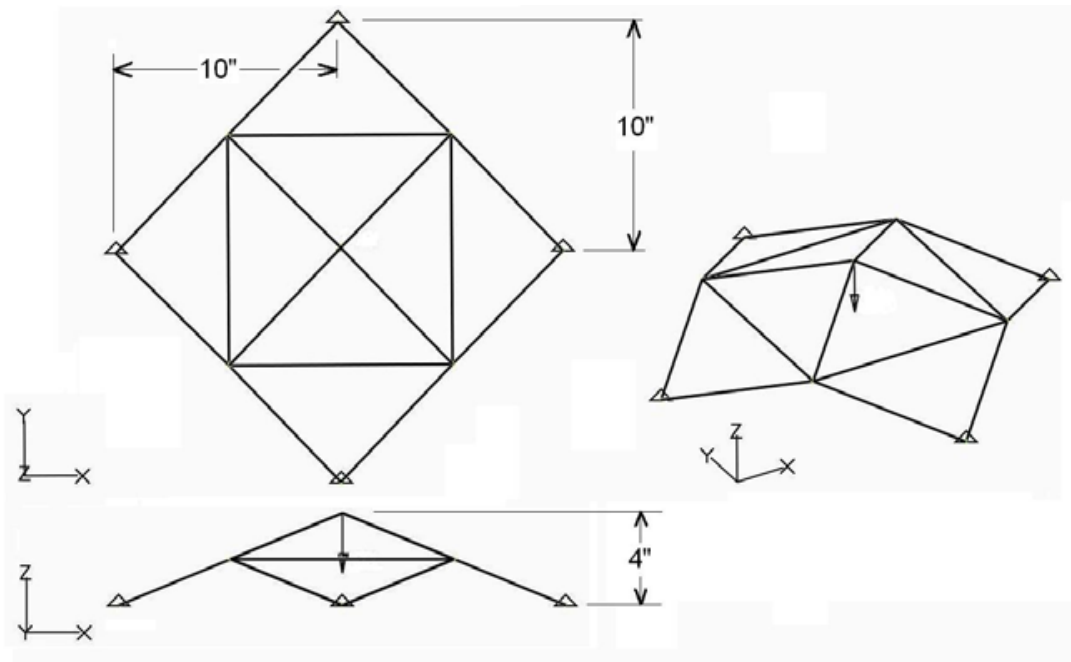

Figure 11. Sixteen-member shallow truss example.

The deformed truss results are shown in Fig. 12. The displacement results for the center node are plotted in Fig. 13; the results indicate several snap-through and snap-back events. In Fig. 13, the complex equilibrium path is shown at turning points with arrows. Table 3 gives the load increments and displacements at several of the turning points. The first limit point, which is shown in Fig. 13, coincides with the first data point that is listed in Table 3 and is the first critical limit point of the structure. The load increment at point 1 is 0.096 and is multiplied by the applied load of $10^{5} \mathrm{lb}$ to equal $9600 \mathrm{lb}$. This product is the maximum load that the structure can support before snap-through buckling. After reaching point 1 , the load increment decreases until another turning point is reached at point 2 . Here, 
the structure is still unstable; however, the load increment reverses and begins to increase. The structure continues to deform until a new stable equilibrium point is reached. This point is where the load increment is equal to the first critical load increment (0.096). In Fig. 13, the second stable equilibrium point is denoted with an $\mathrm{x}$. After the load increment returns to the first critical limit load, the structure becomes static and can support additional load. Point 4 is another equilibrium path turning point. However, after passing this point, the structure begins a sudden snap-back that continues through point 5 and onto point 6 . The equilibrium path resembles a "figure eight" and is a typical shape for structures with snap-back equilibrium paths. The following points, 8 through 10, in Fig. 13 show another snap-through of the structure.

Table 3. Sixteen-Member Shallow Truss

\begin{tabular}{|c|c|c|}
\hline Location & Load Increment & $\begin{array}{c}\text { Displacement, } \\
\text { inches }\end{array}$ \\
\hline 1 & 0.096 & -0.97 \\
\hline 2 & -0.096 & -3.09 \\
\hline 3 & 0.00 & -3.99 \\
\hline 4 & 0.321 & -5.65 \\
\hline 5 & -0.094 & -5.37 \\
\hline 6 & 0.093 & -2.52 \\
\hline 7 & -0.320 & -2.30 \\
\hline 8 & 0.096 & -4.98 \\
\hline 9 & -0.095 & -6.91 \\
\hline 10 & 0.782 & -10.05 \\
\hline
\end{tabular}
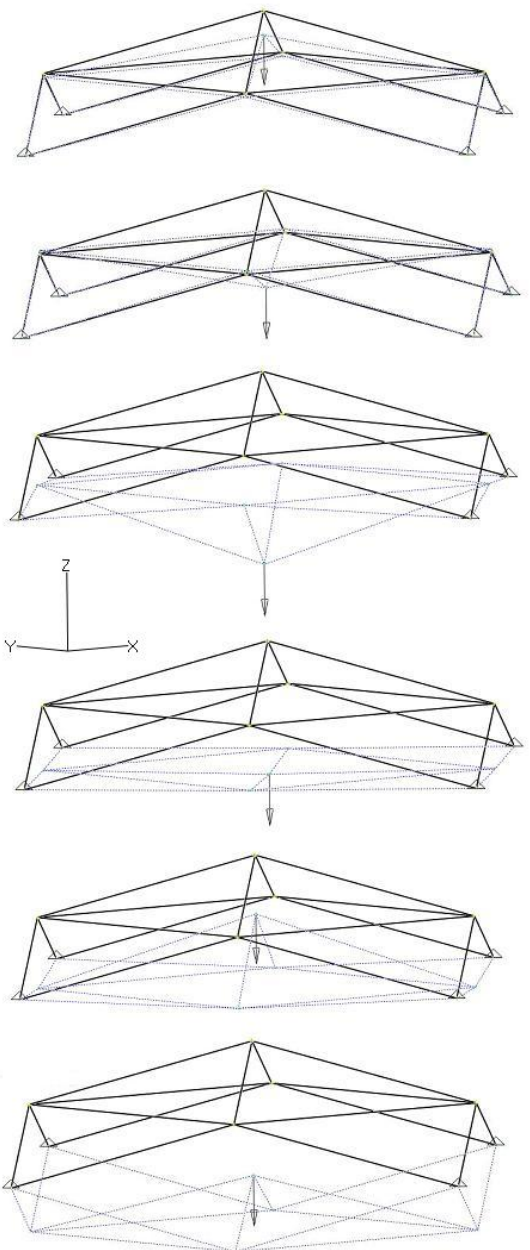

Figure 12. Sixteen-member shallow truss equilibrium path.

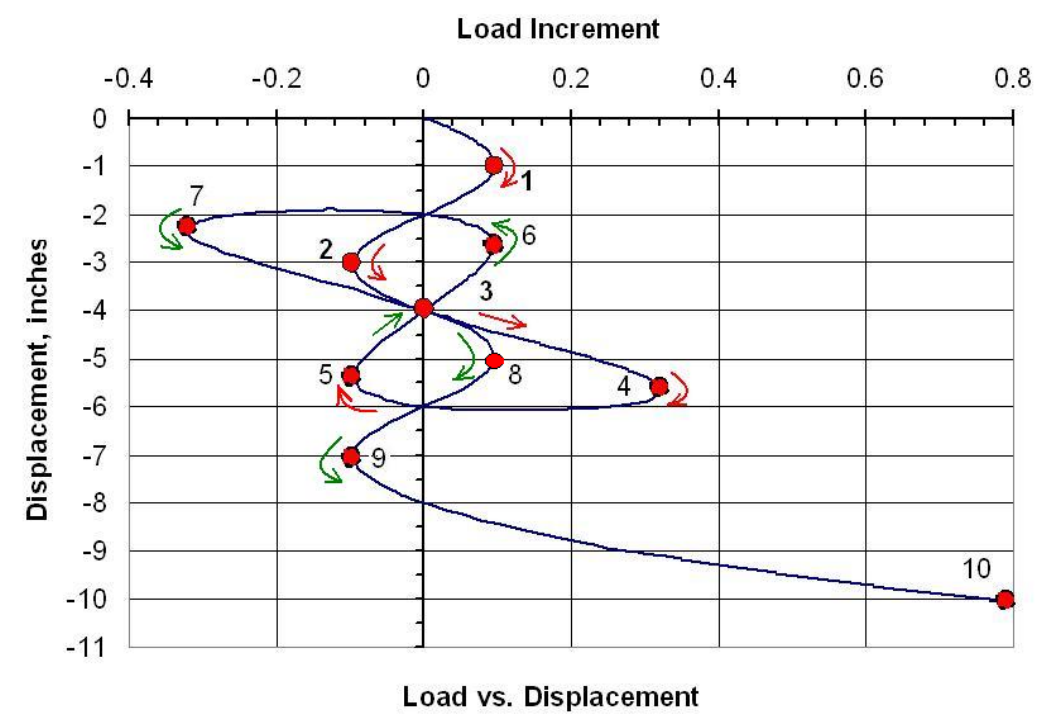

Figure 13. Sixteen-member shallow truss equilibrium path.

American Institute of Aeronautics and Astronautics 


\section{A-Truss}

The fourth snap-through design, which is shown in Fig. 14, is a symmetric A-truss that is assembled with 14 members and 9 nodes. The out-of-plane motion has been constrained with pin supports added to each end of the truss. The cross-sectional area of each member is $1 \mathrm{in}^{2}$ with Young's modulus of $10^{7} \mathrm{lb} / \mathrm{in}^{2}$. The four support nodes are located with a horizontal $x$-axis dimension of 20 in. from the center node and $4 \mathrm{in}$. along the $y$ axis. The center node is raised $4 \mathrm{in}$. along the $z$ axis to complete the shallow truss geometry. As with the previous designs, a vertical load is applied at the center node to investigate buckling. The applied load in this design is $100000 \mathrm{lb}$.
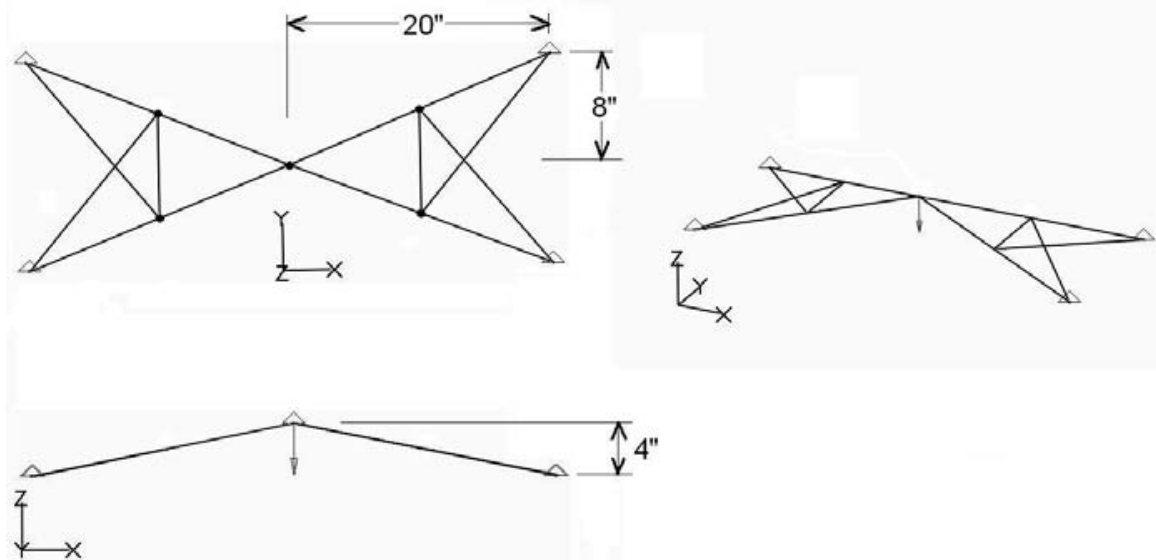

Figure 14. A-truss example.

The deformed geometry is shown in Fig. 15 and captures the snap through. The center node equilibrium path is plotted in Fig. 16 just as in the other examples. The displacement results are obtained from NASTRAN and are plotted to investigate any nonlinear behavior. In the A-truss model, the equilibrium curve has a familiar backward "S" shape that is a typical snap-through curve. The structural response in Fig. 16 shows the critical limit point at a load increment of 0.55 , which corresponds to a $55000 \mathrm{lb}$ load. This is the maximum load that the structure can support while still remaining stable. After point 1 , the structure dynamically snaps through to a second stable equilibrium point. As in the first two examples, a vertical line is drawn on the equilibrium path to intersect point 4 , which is the second stable point in Fig. 16. The structure is now able to support additional loading but will eventually start material yielding.
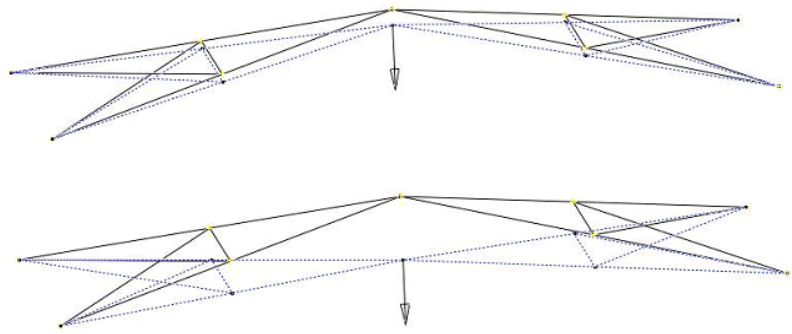

Table 4. A-Truss Example

\begin{tabular}{|c|c|c|}
\hline Location & Load increment & $\begin{array}{c}\text { Displacement, } \\
\text { inches }\end{array}$ \\
\hline 1 & 0.55 & -1.69 \\
\hline 2 & 0.00 & -3.99 \\
\hline 3 & -0.55 & -6.30 \\
\hline 4 & 0.55 & -8.64 \\
\hline
\end{tabular}

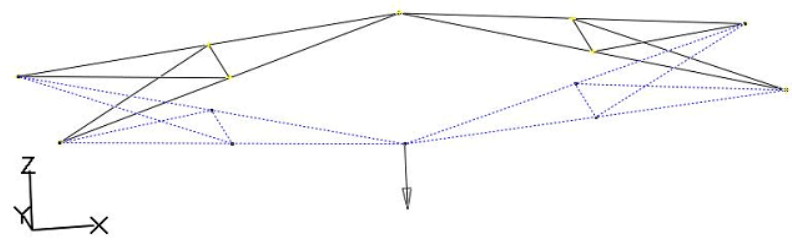

Figure 15. A-truss deformed geometry. 


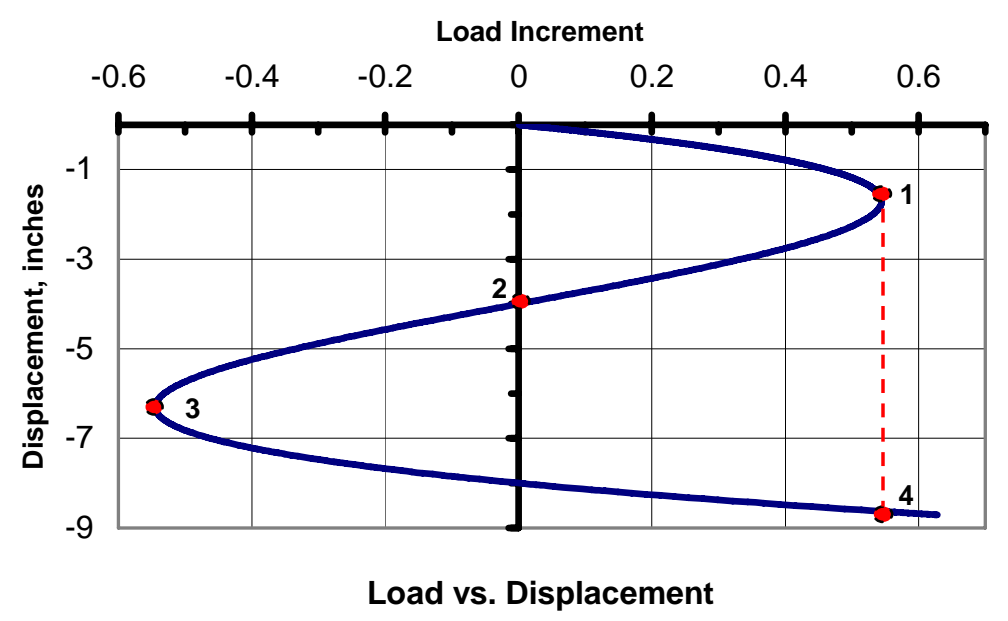

Figure 16. A-truss equilibrium path.

\section{E. Crisfield circular arch truss.}

The model is taken from the second volume of Crisfield ${ }^{8}$ and tests multiple snap-through and snap-back equilibrium paths. The model has 101 elements with 42 nodes and a total of 126 dof. The out-of-plane motion has been constrained with pin supports added to each end of the truss. Fig. 17 shows the finite element model and the applied load of $10^{6} \mathrm{lb}$ at the apex.

The truss deformations are given in Fig. 18 and are similar to those found by Ref. 11. Referring to Fig. 19, the first snap-through, point 1 , starts when the tangent to the equilibrium path is parallel to the displacement axis. The arch becomes unstable and releases stored strain energy and dynamically traces the equilibrium path from 1 to 8 . The deformed structure's vertical apex node values are shown sequentially in Table 5. A snap-back starts at point 3 and continues through 4 and on to 5. The snap-back is still unstable and occurs when the tangent to the equilibrium path is parallel to the load axis. The snap-back continues dynamically until point 6 in Fig. 19 is reached. The equilibrium path now continues through points 7 and 8 where another unstable snap-back begins.

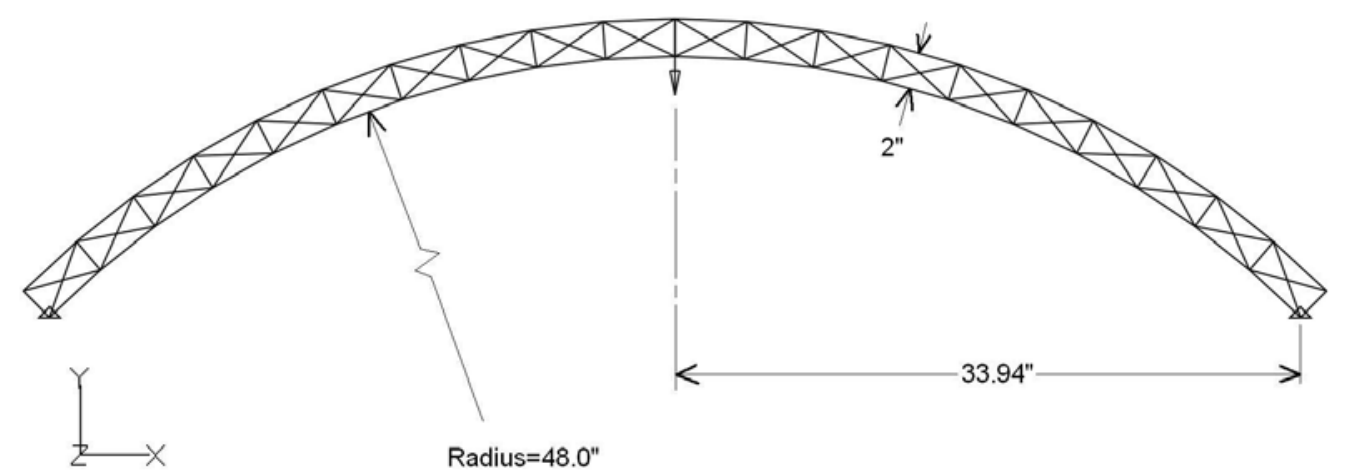

Figure 17. Crisfield arch truss example. 

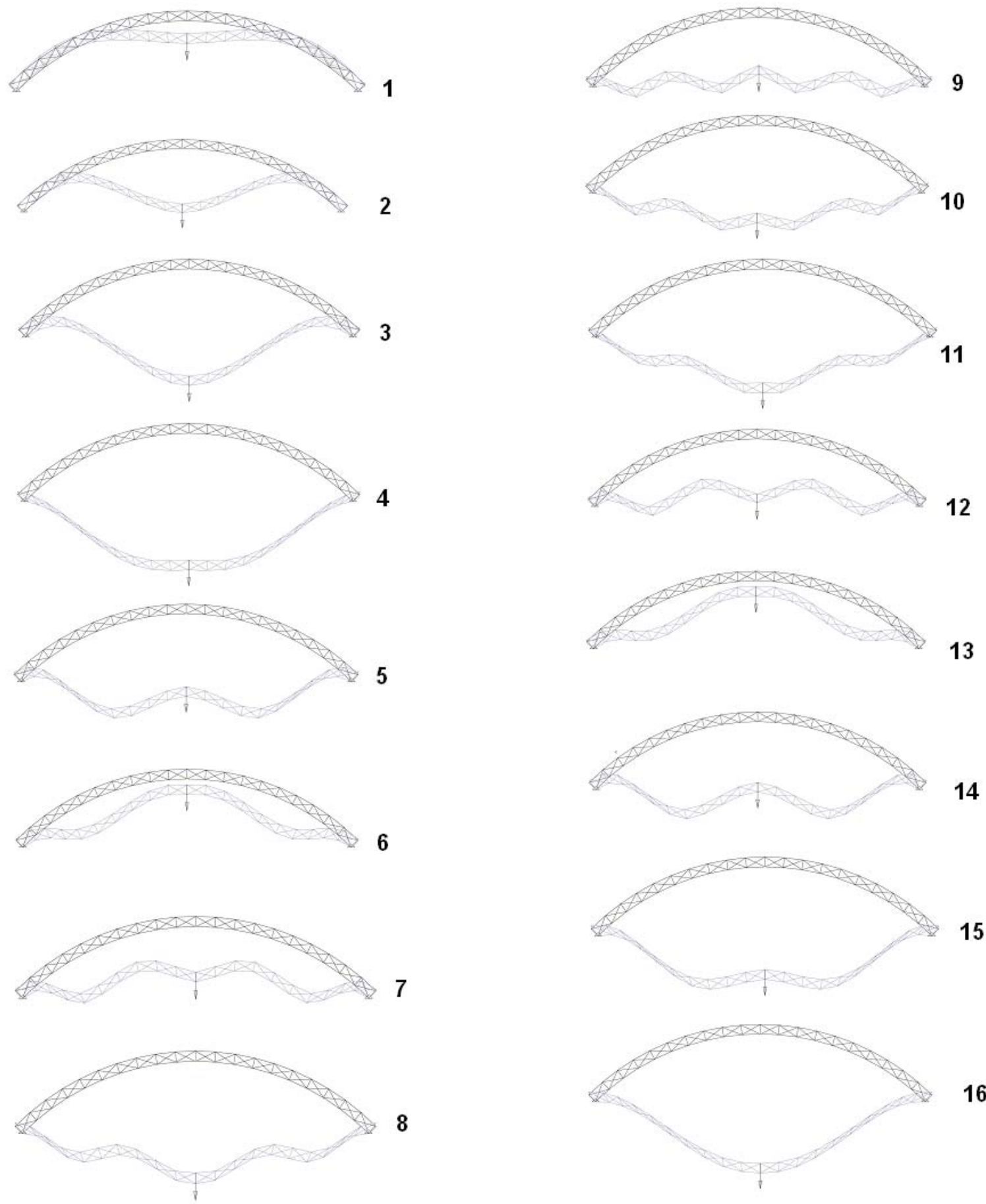

Figure 18. Crisfield arch truss deformed geometry. 


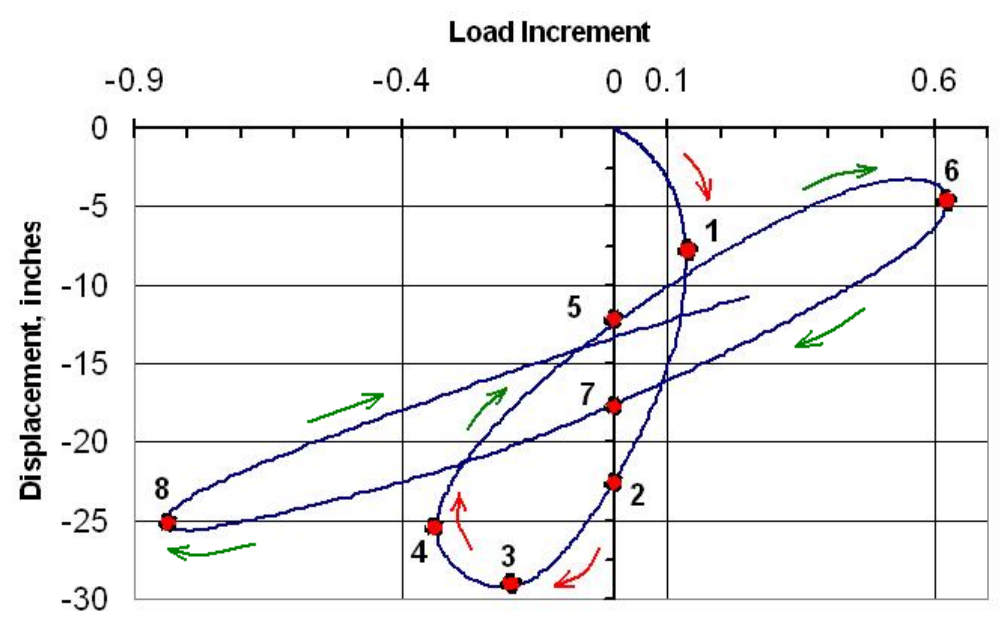

Load vs. Displacement

Figure 19. Arch truss Crisfield equilibrium path.

Table 5. Arch Truss Crisfield Example

\begin{tabular}{|c|c|c|}
\hline Location & Load increment & $\begin{array}{c}\text { Displacement, } \\
\text { inches }\end{array}$ \\
\hline 1 & 0.133 & -7.20 \\
\hline 2 & 0.00 & -22.38 \\
\hline 3 & -0.207 & -29.23 \\
\hline 4 & -0.332 & -25.51 \\
\hline 5 & 0.00 & -12.36 \\
\hline 6 & 0.625 & -4.75 \\
\hline 7 & 0.00 & -17.80 \\
\hline 8 & -0.838 & -24.83 \\
\hline
\end{tabular}

\section{Discussion}

The investigated models displayed snap-through behavior after reaching a critical point. The nonlinear behavior was identified by plotting the equilibrium paths of the center nodes. The nonlinear truss designs were identified by applying different truss geometries until a snap-through occurred; finding a design that kept the snap-through displacement of the center node in the out-of-plane direction was important. The arc length method that is used in the NASTRAN solution allowed the equilibrium path to be followed when the tangent stiffness approached zero. An important feature regarding the appearance of equilibrium paths was identified: an equilibrium path with a backward "S" shape represents a snap-through event. This feature can be seen in the equilibrium paths that are plotted in Fig. 7, 10, and 16, which correspond to designs 1,2 , and 4, respectively. Other observed shapes include the "figure eight" plot that can be seen in Fig.13 and the "flower petal" shape that is evident in Fig. 19. Both of these equilibrium paths are typical for structures that are exhibiting snap-back behavior.

\section{Conclusion}

The exploration of space will require new technologies to enable future missions. New architectures may include platforms that require assembly in space by using a truss system. The safe deployment and assembly of large trusses requires that their nonlinear behavior be well understood through analysis and testing. The work presented in this investigation has contributed to this goal by demonstrating that equilibrium paths can be used to examine nonlinear behavior. Furthermore, the technique that is used here to investigate the snap-through behavior of large trusses may be applied similarly to micro-scaled structures. The miniaturization of trusses is of great interest to the

11

American Institute of Aeronautics and Astronautics 
microelectromechanical systems since miniaturized trusses can be used as rapid switches that can be integrated into solid-state circuits. ${ }^{4}$ One problem in the design of these trusses has been identifying the critical limit point of the switch at snap-through. The truss designs that were investigated in this paper potentially can be micro-sized for incorporation into MEMS devices. These designs represent examples of nonlinear behavior that other researchers or designers may find useful in verifying their analysis techniques.

\section{References}

${ }^{1}$ Pai, P. F., Highly Flexible Structures: Modeling, Computation, and Experimentation, AIAA Education Series, 2007.

${ }^{2}$ Blandford, G. E., "Progressive Failure Analysis of Inelastic Space Truss Structures," Computers and Structures, Vol. 58, 1996, pp. 981-990.

${ }^{3}$ Warren, J. E., Nonlinear Stability Analysis of Frame-Type Structures with Random Geometric Imperfections Using a TotalLagrangian Finite Element Formulation, Ph.D. Dissertation, Virginia Polytechnic Institute and State University, January 1997.

${ }^{4}$ Chollet, F. and Liu, H. B., "A (not so) Short Introduction to Micro Electromechanical Systems", version 3.0, 2009, http://memscyclopedia.org/introMEMS.html.

${ }^{5}$ Doyle, James F., Nonlinear Analysis of Thin-Walled Structures, Springer, West Lafayette, IN, 2001.

${ }^{6}$ Khot, N. S. and Kamat, M. P., "Minimum Weight Design of Truss Structures with Geometric Nonlinear Behavior," AIAA Journal, Vol. 23, No. 1, January 1985.

${ }^{7}$ Crisfield, M. A., Non-Linear Finite Element Analysis of Solids and Structures: Volume I- Essentials, John Wiley \& Sons, London, UK, 1991.

${ }^{8}$ Crisfield, M. A., Non-Linear Finite Element Analysis of Solids and Structures: Volume 2-Advanced Topics, John Wiley \& Sons, London, UK, 1997.

${ }^{9}$ Hrinda, G. A., "Optimization of stability-constrained geometrically nonlinear shallow trusses using an arc length sparse method with a strain energy density approach,” Finite Elements in Analysis and Design, Vol. 30, 2005, pp. 273-296.

${ }^{10}$ Suleman, A. and Sedaghati, R.: "Benchmark Case Studies in Optimization of Geometrically Nonlinear Structures," Structural and Multidisciplinary Optimization, Vol. 30, 2005, pp. 273-296.

${ }^{11}$ Hrinda, G. A., "Geometrically Nonlinear Arc Length Sparse Finite Element Analysis and Optimal Design of Truss Structures,” Ph.D. Dissertation, Civil Engineering Dept., Old Dominion Univ., Norfolk, VA, 2009. 